\title{
On the Distinction of Subjectivity and Objectivity of Emotions in Texts
}

\author{
Manh Hung Nguyen ${ }^{1,2}$ \\ ${ }^{1}$ Posts and Telecommunications Institute of Technology (PTIT) \\ ${ }^{2}$ UMI UMMISCO 209 (IRD/UPMC), Hanoi, Vietnam
}

\begin{abstract}
Emotion classification in texts is an instance of the text classification problem. It therefore could apply some existing text classifiers by considering each emotion as a label of the text. However, most of recent works does not differentiate the subjectivity and objectivity of the same emotion in the text. This paper firstly builds some datasets whose labels are emotion, in which the subject and object of the same emotion are considered as two separated labels. Secondly, this paper evaluates some existing classifiers via some scenarios on the built datasets. The results are then discussed on some difficulties of these kinds of problem.
\end{abstract}

Keywords-Text classification; Emotion classification; subjective emotion; objective emotion

\section{INTRODUCTION}

Emotion classification in text (or emotion recognition from text) is one of popular instances of the text classification in general. It could be applied into several applications. For instances, in the application of consumer sentiment analysis where the detection of emotion in the consumer feedback may help other consumer to choose the best seller or help sellers to improve their services. In the application of home-machine interaction where the computer and the user can take some conversations, recognition of user emotion in the conversation text may help the computer to improve the effect of the conversation. In the application of measurement the similarity among social network users, recognition of emotion in the posts (status), or the comments of users on some other's posts could help us to measure the similarity among users' opinion on the topic. These data may then help us to further analyse and/or predict the similarity of these users on their interests or their behavior of online shopping, etc.

As this is an instance of the text classification problem, it thus could use some existing (and popular) text classifiers. However, most of recent works does not differentiate the subjectivity and objectivity of the same emotion in the text. For instances, let's consider these two following texts:

- Text A: I am happy

- $\quad$ Text B: They are happy, but me not

Most of current text classifiers will assign both these texts to the label of joy because they do not differentiate the happiness of the teller (subjective joy), and that of other (objective joy). Meanwhile, in the case of emotion classification with the distinction of their subjectivity/objectivity, these two texts may be assigned to different labels: the text A may be assigned to the label of subjective joy, and the text B may assigned to the labels objective joy and subjective sadness. Intuitively, the case of emotion classification with the distinction of their subjectivity/objectivity could classify the texts more precisely than the case without distinction of emotion subjectivity/objectivity. But the second case may be more difficult than the first case.

This paper investigates in the problem of emotion classification in texts with the distinction of their subjectivity/objectivity. This paper firstly builds some datasets whose labels are emotion, in which the subject and object of the same emotion are considered as two separated labels. Secondly, this paper evaluates some existing classifiers via some scenarios on the built datasets. The results are then discussed on some difficulties of these kinds of problem.

This paper is organised as follows: Section II presents some related works. Section III presents the build of datasets of texts labelled with subjective/objective emotions. Section IV presents some preliminary experiments on the built datasets. Finally, section $\mathrm{V}$ is a conclusion.

\section{RELATED WORKS}

Researches on emotion in computer science domain are mainly based on the appraisal and cognitive theories of emotions such as the cognitive structure of emotion of Ortony et al. [29], the cognitive pattern of emotion of Lazarus [13], [19] and the belief-desire theory of emotion (BDTE) of Reisenzein [34]. These attempts could be regrouped into three mains directions. First, the approaches to represent the concept of emotions (Van Dyke Parunak et al. [30], and Stephane [37]), to formalize some emotions in a formal logic (Meyer [21], Ochs et al. [28], and Bonnefon et al. [5], [6], [25]), and to calculate the degree of emotions (Steunebrink et al. [38], Nguyen [26]). This direction is far from our work, therefore this paper does not investigate in this direction.

Second, the approaches to recognize the emotion from facial expression (Ekman [11], Russe [36], Adolphs [1], and Busso et al. [9]). This direction is also far from our work, therefore this paper does not investigate in this direction.

Third, the approaches to recognize the emotion from text. This is an instance of the problem of text classification. Therefore, we can use any method of text classification in to the application of emotion detection. For instance, we can use any of (or extended of any ) classical classifiers such as Naive Bayes (NB) [16], Support Vector Machine (SVM) [8], k-Nearest Neighbors (kNN or IBk) [2], C4.5 [33]. Moreover, some authors could improve some classical classifier for their model. For instances, Danesh et al. proposed three improvements using Decision Template, or Voting, or 
Ordered Weighted Averaging (OWA) [10]; Erkan et al. [12] proposed a model with a Harmonic function; Nigam et al. [27] proposed the expectationmaximization (EM); Kibriya et al. [17] proposed the Multinomial Naive Bayes.

Recently, some authors proposed their model for emotion detection in text. For instances, Alm et al. [3] used supervised machine learning with the SNoW learning architecture. Szpakowicz and colleagues [4], [14] used annotation scheme. Li and $\mathrm{Xu}[20]$ tried to infer and extract the reasons of emotions by importing knowledge and theories from other fields such as Sociology. Kralj et al. [18] investigated in EMOJIS, emotion expression in Twitter. Perikos and Hatzilygeroudis [31] used an ensemble of classifier: two are statistical (a Nave Bayes and a Maximum Entropy learner) and the third one is a knowledgebased tool performing deep analysis of the natural language sentences.

However, most of these models are not tested with the subjectivity/objectivity of the same emotion. Therefore, this paper aims to evaluate some existing classifiers on the problem of emotion classification with the distinction of their subjectivity/objectivity.

\section{DATASET}

This section presents some related datasets for the problem of emotion classification; and then, builds some datasets which support the classification of subjective/objective emotions.

\section{A. Related datasets}

There are many datasets built for the problem of emotion classification. These datasets could be divided into two groups. Firstly, group of single label, in which a text has only one label. For instances, Plutchik [32], CrowdFlower [24], EmoLex [23], and Semeval2017 [22]. Secondly, group of multi-label, in which a text may have more than one label. For instance, Brat data [7]. These are presented in Table.I.

TABLE I. Some Related EMotion DATASETS

\begin{tabular}{|l|c|c|c|c|}
\hline Dataset & $\begin{array}{c}\text { N. } \\
\text { sample }\end{array}$ & $\begin{array}{c}\text { N. } \\
\text { emotion }\end{array}$ & single/multi & Sub/obj \\
\hline Brat data [7] & 629 & 8 & multi & no \\
Semeval2017 [22] & 6755 & 4 & single & no \\
EmoLex [23] & 20000 & 6 & single & no \\
CrowdFlower [24] & 40000 & 13 & single & no \\
Plutchik [32] & 2524 & 19 & single & no \\
\hline
\end{tabular}

However, most of related datasets do not support the distinction of subjectivity and objectivity of emotion. In these datasets, the default label is that subjective emotion. Therefore, these datasets could not be used for the problem of emotion classification with the distinction of their subjectivity/objectivity. This is the main reason this paper has to build some new datasets to support this problem.

\section{B. Built dataset}

In order to build some dataset for the problem of emotion classification with the distinction of their subjectivity/objectivity, we collected several texts from several sources: status on social networks, title of news papers, idioms and quotations, lyric of songs, etc. These texts are then labelled with (subjective and/or objective) emotions. The emotions are mainly based on the cognitive definition of Frijda [13] and Lazarus [19]. The texts are divided into two dataset based on language: Vietnamese and English.

TABLE II. Distribution of EMotion Number In Two Datasets

\begin{tabular}{|l|c|c|}
\hline \multirow{2}{*}{ Emotion } & \multicolumn{2}{|c|}{ Number of text } \\
\cline { 2 - 3 } & dataset VN & dataset EN \\
\hline 1 emotion & 849 & 481 \\
2 emotions & 547 & 307 \\
3 emotions and more & 104 & 12 \\
\hline All & $\mathbf{1 5 0 0}$ & $\mathbf{8 0 0}$ \\
\hline
\end{tabular}

TABLE III. Distribution of EMotion Type in Two Datasets

\begin{tabular}{|l|c|c|c|c|c|c|}
\hline \multirow{2}{*}{ Emotion } & \multicolumn{3}{|c|}{ dataset VN } & \multicolumn{3}{c|}{ dataset EN } \\
\cline { 2 - 7 } & Sub. & Obj. & All & Sub. & Obj. & All \\
\hline Joy & 81 & 86 & 167 & 57 & 58 & 115 \\
Sadness & 162 & 116 & 278 & 61 & 66 & 127 \\
Hope & 54 & 50 & 104 & 25 & 25 & 50 \\
Fear & 110 & 88 & 198 & 37 & 34 & 71 \\
Satisfaction & 48 & 53 & 101 & 24 & 26 & 50 \\
Disappointment & 93 & 62 & 155 & 30 & 31 & 61 \\
Love & 58 & 65 & 123 & 27 & 24 & 51 \\
Disgust & 161 & 100 & 261 & 26 & 24 & 50 \\
Pride & 48 & 71 & 119 & 23 & 24 & 47 \\
Shame & 59 & 56 & 115 & 25 & 24 & 49 \\
Admiration & 56 & 55 & 113 & 22 & 29 & 51 \\
Gratitude & 45 & 48 & 93 & 24 & 24 & 48 \\
Anger & 71 & 67 & 138 & 24 & 24 & 48 \\
Other & 153 & 174 & 327 & 148 & 164 & 312 \\
\hline All & $\mathbf{1 1 9 9}$ & $\mathbf{1 0 9 3}$ & $\mathbf{2 2 9 2}$ & $\mathbf{5 5 3}$ & $\mathbf{5 7 7}$ & $\mathbf{1 1 3 0}$ \\
\hline
\end{tabular}

In the Vietnamese dataset, there are about 1500 samples. Meanwhile the English dataset has about 800 samples. The distribution of samples on each label is presented in the Table.III. And the distribution of samples on the number of label for each sample is presented in the Table.II. These are multi-label datasets: each text may have more than one label.

One of the most important feature of these two datasets is that in their labels, the subjectivity and the objectivity of the emotion are distinguished. A text may have only a subjective emotion, or objective emotion, or both subjective and objective of an emotion. For instances, the text "My dream becomes true!" may have two labels of subjective satisfaction and subjective joy. Meanwhile, the text "His dream becomes true, but not mine!" may have four labels: objective satisfaction, objective joy (for him), subjective disappointment, and subjective sadness (for the teller). In this case, the subjective satisfaction and objective satisfaction are considered as two different labels. Therefore, in these two datasets, there are only 14 different emotions, but there are 28 different labels because of the distinction of their subjectivity/objectivity.

\section{EVALUATION}

\section{A. Experiment 1: Evaluation of the classifiers}

The objective of this experiment is to find out the most suitable classifier for these datasets. The found classifier will be used in the next experiments.

1) Scenario: This experiment is taken with the following scenario for each dataset:

1 For each text in the dataset, remove all stop-words.

2 Split the remain character sequence into 1-gram.

3 Transform it into a vector of TF-IDF value. 
$4 \quad$ Using the k-folds crossed-validation: Split the dataset into ten sets (10-folds). Each time, a set is used for testing (called testing set), and the nine remain sets are used for training (called training set).

5 Train and test with following classifiers ${ }^{1}$ :

5.1 Support Vector Machine (SVM) [8].

5.2 K-Nearest Neighbors (KNN or IBk) [2].

5.3 C4.5 (J48) [33].

5.4 Rotation Forest (RF) [35].

5.5 Naive Bayes (NB) [16].

5.6 Multinomial Naive Bayes (MNB) [17].

6 Note the observed output parameters for each time of running.

7 Repeat the steps from 5 to 6 in ten times (10-folds) and take the mean values of each output parameters for all times of running.

2) Output parameters: Let's $O_{i}$, and $E_{i}$ are respectively the original set of label and the extracted set of label of the text $i$. And $C_{i}=O_{i} \cap E_{i}$ is the intersection set of $O_{i}$ and $E_{i}$. We make use of these parameters:

- $\quad$ The precision on the sample $i$ is:

$$
\text { pre }_{i}=\frac{\left|C_{i}\right|}{\left|E_{i}\right|} * 100 \%
$$

- $\quad$ The precision on all $n$ samples in the test set is:

$$
\text { Precision }=\frac{\sum_{i=1}^{n} \text { pre }_{i}}{n}=\frac{1}{n} * \sum_{i=1}^{n} \frac{\left|C_{i}\right|}{\left|E_{i}\right|} * 100 \%
$$

- The recall on the sample $i$ is:

$$
r e c_{i}=\frac{\left|C_{i}\right|}{\left|O_{i}\right|} * 100 \%
$$

- $\quad$ The recall on all $n$ samples in the test set is:

$$
\text { Recall }=\frac{\sum_{i=1}^{n} \text { rec }_{i}}{n}=\frac{1}{n} * \sum_{i=1}^{n} \frac{\left|C_{i}\right|}{\left|O_{i}\right|} * 100 \%
$$

- The F1-score on all samples of the test set is:

$$
F 1-\text { score }=\frac{2 * \text { Precision } * \text { Recall }}{(\text { Precision }+ \text { Recall })}
$$

For each experiment, we consider the results on three output parameters: Precision, Recall, and F1-score.

TABLE IV. COMparison Results Among Some Classifiers

\begin{tabular}{|l|c|c|c|c|c|c|}
\hline \multirow{2}{*}{ Classifier } & \multicolumn{3}{|c|}{ dataset VN } & \multicolumn{3}{c|}{ dataset EN } \\
\cline { 2 - 7 } & Pre. & Rec. & F1. & Pre. & Rec. & F1. \\
\hline SVM & 0.00 & 0.00 & 0.00 & 0.00 & 0.00 & 0.00 \\
C4.5 (J48) & 2.50 & 1.63 & 1.94 & 0.45 & 0.40 & 0.40 \\
RF & 2.50 & 1.63 & 1.94 & 0.55 & 0.50 & 0.50 \\
KNN & 17.41 & 18.44 & 17.89 & 10.28 & 12.28 & 11.11 \\
NB & 17.70 & 25.77 & 20.91 & 13.76 & 22.24 & 16.96 \\
MNB & 24.89 & 44.54 & 31.86 & 16.64 & 35.50 & 22.62 \\
\hline
\end{tabular}

\footnotetext{
${ }^{1}$ These classifiers are called from API of Weka open source library [39], [15] for Java.
}

3) Results: The results are presented in the Table.IV: The classifier SVM gets the lowest value on all three output parameters, on both datasets. KNN gets higher value than SVM; C4.5 and RF get higher value than SVM; KNN gets higher value than $\mathrm{C} 4.5$; NB gets higher value than KNN. And the classifier MNB gets the highest value on all three output parameters, on both datasets. Therefore, the MNB is the chosen classifier for the next experiments.

\section{B. Experiment 2: The effects of the stop-words}

The objective of this experiment is to test the effect of stopwords on the distinguish of subjective and objective emotion in texts. Therefore, this experiment will compare two strategies in pre-processing of data: remove (without) or not remove (with) stop-words from the texts.

1) Scenario: This experiment is taken with the following scenario for each dataset:

1 For each text in the dataset, consider two cases:

$$
\begin{aligned}
& \text { 1.1 All stop-words are removed. } \\
& 1.2 \text { Do not remove stop-words. }
\end{aligned}
$$

2 Split the remain character sequence into 1-grams.

3 Transform each text into a vector of TF-IDF value.

4 Using the k-folds crossed-validation: Split the dataset into ten sets (10-folds). Each time, a set is used for testing, and the nine remain sets are used for training.

5 Train and test with the classifier of Multinomial Naive Bayes (MNB).

6 Note the observed output parameters for each time of running.

7 Repeat the step from 5 to 6 in ten times (10-folds) and take the mean values of each output parameters for all times of running.

In this experiment, three output parameters are also used: Precision, Recall, and F1-score.

TABLE V. COMParison Results In the CASE Without AND With STOP-WORD

\begin{tabular}{|l|c|c|c|c|c|c|}
\hline \multirow{2}{*}{ Method } & \multicolumn{3}{|c|}{ dataset VN } & \multicolumn{3}{c|}{ dataset EN } \\
\cline { 2 - 7 } & Pre. & Rec. & F1. & Pre. & Rec. & F1. \\
\hline without stop-word & 24.89 & 44.54 & 31.86 & 16.64 & 35.50 & 22.62 \\
with stop-word & 25.68 & 44.44 & 32.50 & 18.76 & 32.88 & 23.80 \\
\hline
\end{tabular}

2) Results: The results are presented in the Table.V on three output parameters, on both datasets. At the level of precision, the value in the case with stop-words is higher than that in the case without stop-words, on both datasets. Meanwhile, at the level of recall, the value in the case with stop-words is lower than that in the case without stop-words, on both datasets. However, at the level of F1-score, the value in the case with stop-words is higher than that in the case without stop-words, on both datasets.

Based on these results, in the next experiment, all stopwords are not removed from the texts. 


\section{Experiment 3: The effects of $N$-gram}

The objective of this experiment is to find out the best $n$ in the n-gram extraction of texts. This experiment will consider five gram-extraction strategies: using only 1-gram, from 1 to 2 -grams, from 1 to 3 -grams, from 1 to 4 -grams, and from 1 to 5-grams.

1) Scenario: This experiment is taken with the following scenario for each dataset:

1 For each text in the dataset, remove all stop-words.

2 Each time, using one of these five following gramextraction strategies:

2.1 1-gram: using only 1-gram.

2.2 From 1 to 2-grams: using both 1-gram and 2grams.

2.3 From 1 to 3-grams: using 1-gram, 2-grams, and 3-grams.

2.4 From 1 to 3-grams: using 1-gram, 2-grams, 3grams, and 4-grams.

2.5 From 1 to 3-grams: using 1-gram, 2-grams, 3grams, 4-grams, and 5-grams.

3 Transform each text into a vector of TF-IDF value.

4 Using the k-folds crossed-validation: Split the dataset into ten sets (10-folds). Each time, a set is used for testing, and the nine remain sets are used for training.

5 Train and test with the classifier of Multinomial Naive Bayes (MNB).

6 Note the observed output parameters for each time of running.

7 Repeat the step from 5 to 6 in ten times (10-folds) and take the mean values of each output parameters for all times of running.

In this experiment, three output parameters are also used: Precision, Recall, and F1-score.

TABLE VI. COMPARISON RESULTS AMONG DIFFERENT N-GRAMS

\begin{tabular}{|l|c|c|c|c|c|c|}
\hline \multirow{2}{*}{ N-gram } & \multicolumn{3}{|c|}{ dataset VN } & \multicolumn{3}{c|}{ dataset EN } \\
\cline { 2 - 7 } & Pre. & Rec. & F1. & Pre. & Rec. & F1. \\
\hline 1-gram & 18.76 & 32.88 & 23.80 & 25.68 & 44.44 & 32.50 \\
1 to 2-grams & 21.55 & 30.40 & 25.17 & 31.82 & 51.15 & 39.20 \\
1 to 3-grams & 20.74 & 32.48 & 25.27 & 33.70 & 50.69 & 40.45 \\
1 to 4-grams & 21.67 & 30.76 & 25.41 & 34.40 & 49.69 & 40.61 \\
1 to 5-grams & 21.85 & 31.21 & 25.65 & 35.59 & 48.56 & 41.02 \\
\hline
\end{tabular}

2) Results: The results are presented in the Table.VI: Generally, the higher the n-gram is up to, the higher the value of output parameters, in both datasets. However, from the value of 3-grams, the increment of output parameters is slowdown and there is no significant difference among three output parameters in the case of 3-grams, 4-grams, and 5grams. Therefore, it is sufficient to use the case up to 3-grams.

\section{Experiment 4: The difficulty of the problem}

This experiment will compare the case of emotion classification with or without distinction of their subjectivity/objectivity to see how hard the problem of emotion classification with distinction of their subjectivity/objectivity in comparing to the classical problem of emotion classification without distinction of their subjectivity/objectivity.
1) Scenario: This experiment is taken with the following scenario for each dataset:

1 For each text in the dataset, do not remove stop-words.

2 Split the remain character sequence into grams from 1 to 3 -grams.

3 Transform each text into a vector of TF-IDF value.

4 Using the k-folds crossed-validation: Split the dataset into ten sets (10-folds). Each time, a set is used for testing, and the nine remain sets are used for training.

5 Train and test with the classifier of Multinomial Naive Bayes (MNB) in three cases:

5.1 Emotion only: Only emotions are differentiated. It means that the subjective and objective of an emotion are considered as the same label, that is the given emotion. For example, subjective joy and objective joy are considered as the same label of joy. So in this case, there are only 14 labels to classify.

5.2 Subjectivity/objectivity only: Only the subjectivity and objectivity of emotion are differentiated. It means that the subjective of all emotions are considered as only one label, the same for objectivity. For example, subjective joy, subjective disappointment, and subjective anger are considered as the same label of subjective. So in this case, there are only two labels to classify (subjective and objective).

5.3 Emotion + subjectivity/objectivity: This is the original problem of this paper. Each of 14 emotions are differentiated in their subjectivity/objectivity. So in this case, there is totally 28 labels to classify.

6 Note the observed output parameters for each time of running.

7 Repeat the step from 5 to 6 in ten times (10-folds) and take the mean values of each output parameters for all times of running.

In this experiment, three output parameters are also used: Precision, Recall, and F1-score.

TABLE VII. COMParison Results Among Different Problems

\begin{tabular}{|l|c|c|c|c|c|c|}
\hline \multirow{2}{*}{ Problem } & \multicolumn{3}{|c|}{ dataset VN } & \multicolumn{3}{c|}{ dataset EN } \\
\cline { 2 - 7 } & Pre. & Rec. & F1. & Pre. & Rec. & F1. \\
\hline Emotion only & 38.05 & 49.16 & 42.85 & 52.28 & 65.29 & 58.03 \\
Sub/obj only & 65.40 & 67.30 & 66.31 & 72.44 & 73.31 & 72.86 \\
Emotion+sub/obj & 20.74 & 32.48 & 25.27 & 33.70 & 50.69 & 40.45 \\
\hline
\end{tabular}

2) Results: The results are presented in the Table.VII on three output parameters. Unsurprisingly, the output values of the first problem, classification of emotion without distinction of their subjectivity/objectivity (classification of 14 labels), are much higher than those in the third problem, classification emotion with distinction of subjectivity/objectivity (classification of 28 labels), on both datasets. And the output values of the second problem, classification of the subjectivity/objectivity only (classification of 2 labels), are much higher than those in the first problem (classification of 14 labels), on both datasets. 
There are two reasons to be considered. Firstly, in the case of emotion classification with the distinction of subjectivity/objectivity, the number of label is double than in the case of classical emotion classification. Generally, in the context of classification problem, the higher the number of label to classify, the more difficult the problem.

Secondly, that is the difficulty of the differentiation between the two label of the same emotion, but different subjective/objective. Let's return to the example from the introduction section:

- Text A: I am happy

- Text B: They are happy, but me not

In the case of emotion classification without subjectivity/objectivity, it could be easy to detect that both these two texts are in the label of joy. However, in the case with the distinction of subjectivity/objectivity, the results are totally different: the texts A belongs to the label of subjective joy, meanwhile the texts B belongs to two labels objective joy and subjective sad. We can see the difficulty of classify these texts among two labels subjective joy and objective joy.

These results indicate that the problem of classification emotion with distinction of subjectivity/objectivity is much more difficult than the classical classification of emotion without distinction of their subjectivity/objectivity. Consequently, the current classifiers could not reach an average value for output parameters. Meanwhile they could get an above-average value when applying them into problem of classification of emotion without distinction of their subjectivity/objectivity. This could be considered as a challenge for researches in the near futures.

\section{CONCLUSION}

This paper considered the problem of emotion classification with the distinction of their subjectivity/objectivity. There are two datasets of text labelled with subjective/objective emotions are built and introduced, one in English, another in Vietnamese. This paper also taken some very preliminary experiments to evaluate some current statistical-based classifiers on these kind of problem. The results indicate that there are two different aspects regarding the classical emotion classification problem (without distinction of subjectivity/objectivity): first, using stop-words is better for differentiating the subjectivity and the objectivity of emotion in texts. Secondly, using current statistical-based classifiers such as SVM, KNN, C4.5, NB, RF, MNB could no more helpful in the given problem.

These difficulties are our challenges and objectives to work in the near future.

\section{REFERENCES}

[1] Ralph Adolphs. Recognizing emotion from facial expressions: Psychological and neurological mechanisms. Behavioral and Cognitive Neuroscience Reviews, 1(1):21-62, 2002.

[2] D. Aha and D. Kibler. Instance-based learning algorithms. Machine Learning, 6:37-66, 1991.

[3] Cecilia Ovesdotter Alm, Dan Roth, and Richard Sproat. Emotions from text: Machine learning for text-based emotion prediction. In Proceedings of the Conference on Human Language Technology and Empirical Methods in Natural Language Processing, HLT '05, pages 579-586, Stroudsburg, PA, USA, 2005. Association for Computational Linguistics.
[4] Saima Aman and Stan Szpakowicz. Identifying expressions of emotion in text. In Proceedings of the 10th International Conference on Text, Speech and Dialogue, TSD'07, pages 196-205, Berlin, Heidelberg, 2007. Springer-Verlag.

[5] Jean-François Bonnefon, Dominique Longin, and Manh Hung Nguyen. Relation of trust and social emotions: A logical approach. In Web Intelligence and Intelligent Agent Technologies, 2009. WI-IAT'09. IEEE/WIC/ACM International Joint Conferences on, volume 2, pages 289-292. IET, 2009.

[6] Jean-François Bonnefon, Dominique Longin, and Manh Hung Nguyen. A logical framework for trust-related emotions. Electronic Communications of the EASST, 22:1-15, 2010.

[7] Lars Buitinck, Jesse van Amerongen, Ed Tan, and Maarten de Rijke. Multi-emotion detection in user-generated reviews. In Proc. European Conference on Information Retrieval (ECIR), 2015.

[8] Christopher J. C. Burges. A tutorial on support vector machines for pattern recognition. Data Mining and Knowledge Discovery, 2(2):121167, jun 1998.

[9] Carlos Busso, Zhigang Deng, Serdar Yildirim, Murtaza Bulut, Chul Min Lee, Abe Kazemzadeh, Sungbok Lee, Ulrich Neumann, and Shrikanth Narayanan. Analysis of emotion recognition using facial expressions, speech and multimodal information. In Proceedings of the 6th International Conference on Multimodal Interfaces, ICMI '04, pages 205-211, New York, NY, USA, 2004. ACM.

[10] A. Danesh, B. Moshiri, and O. Fatemi. Improve text classification accuracy based on classifier fusion methods. In Information Fusion, 2007 10th International Conference on, pages 1-6, July 2007.

[11] Paul Ekman. Facial expressions of emotion: New findings, new questions. Psychological Science, 3(1):34-38, 1992.

[12] Gunes Erkan, Ahmed Hassan, Qian Diao, and Dragomir R Radev. Improved nearest neighbor methods for text classification. Technical report, Technical Report CSE-TR-576-11, University of Michigan. Department of Electrical Engineering and Computer Science, 2011.

[13] Nico H. Frijda. The Emotions: Studies in Emotion \& Social Interaction. Edition de la Maison des Sciences de l'Homme. Cambridge University Press, Paris, 1986.

[14] Diman Ghazi, Diana Inkpen, and Stan Szpakowicz. Hierarchical versus flat classification of emotions in text. In Proceedings of the NAACL HLT 2010 Workshop on Computational Approaches to Analysis and Generation of Emotion in Text, CAAGET '10, pages 140-146, Stroudsburg, PA, USA, 2010. Association for Computational Linguistics.

[15] Mark Hall, Eibe Frank, Geoffrey Holmes, Bernhard Pfahringer, Peter Reutemann, and Ian H. Witten. The weka data mining software: An update. SIGKDD Explorations Newsletter, 11(1):10-18, November 2009.

[16] George H. John and Pat Langley. Estimating continuous distributions in bayesian classifiers. In Eleventh Conference on Uncertainty in Artificial Intelligence, pages 338-345, San Mateo, 1995. Morgan Kaufmann.

[17] Ashraf M. Kibriya, Eibe Frank, Bernhard Pfahringer, and Geoffrey Holmes. Multinomial naive bayes for text categorization revisited. In Proceedings of the 17th Australian Joint Conference on Advances in Artificial Intelligence, AI'04, pages 488-499, Berlin, Heidelberg, 2004. Springer-Verlag.

[18] Petra Kralj Novak, Jasmina Smailovic, Borut Sluban, and Igor Mozetic?. Sentiment of emojis. PLOS ONE, 10(12):1-22, 122015.

[19] Richard S. Lazarus. Emotion \& Adaptation. Oxford University Press, 1991.

[20] Weiyuan Li and Hua Xu. Text-based emotion classification using emotion cause extraction. Expert Syst. Appl., 41(4):1742-1749, March 2014.

[21] John-Jules Ch. Meyer. Reasoning about emotional agents: Research articles. International Journal of Intelligent Systems, 21(6):601-619, 2006.

[22] Saif M. Mohammad and Felipe Bravo-Marquez. Emotion intensities in tweets. In Proceedings of the sixth joint conference on lexical and computational semantics (*Sem), Vancouver, Canada, 2017.

[23] Saif M. Mohammad and Svetlana Kiritchenko. Using hashtags to capture fine emotion categories from tweets. Computational Intelligence, 31(2):301-326, 2015. 
[24] Saif M. Mohammad and Peter D. Turney. Crowdsourcing a wordemotion association lexicon. Computational Intelligence, 29(3):436465, 2013.

[25] Manh Hung Nguyen. A logical framework for Trust-Related emotions: formal and behavioral results. $\mathrm{PhD}$ thesis, University of Toulouse III, 2010.

[26] Manh Hung Nguyen. Combination of cognitive and quantitative aspects to estimate the degree of emotions. Journal of Computer Science and Cybernetics, 32(3):209-224, 2016.

[27] Kamal Nigam, Andrew Kachites Mccallum, Sebastian Thrun, and Tom Mitchell. Text classification from labeled and unlabeled documents using em. Machine Learning, 39(2):103-134, May 2000.

[28] Magalie Ochs, Karl Devooght, David Sadek, and Catherine Pelachaud. A computational model of capability-based emotion elicitation for rational agent. In Dirk Reichardt, Paul Levi, and John-Jules C. Meyer, editors, Proceedings of the 1st workshop on Emotion and Computing Current Research and Future Impact, Bremen, Germany, June 2006.

[29] Andrew Ortony, Geraldl L. Clore, and Allan Collins. The Congnitive Structure of Emotions. The Cambridge University Press, 1988.

[30] H. Van Dyke Parunak, Robert Bisson, Robert Matthews Sven Brueckner, and John Sauter. A model of emotions for situated agents. AAMAS'06, pages 993-995, May 2006.

[31] Isidoros Perikos and Ioannis Hatzilygeroudis. Recognizing emotions in text using ensemble of classifiers. Eng. Appl. Artif. Intell., 51(C):191201, May 2016.
[32] Robert Plutchik. A psychoevolutionary theory of emotions. Social Science Information, 21(4-5):529-553, 1982.

[33] Ross Quinlan. C4.5: Programs for Machine Learning. Morgan Kaufmann Publishers, San Mateo, CA, 1993.

[34] Rainer Reisenzein. Emotions as metarepresentational states of mind: Naturalizing the belief-desire theory of emotion. Cognitive Systems Research, 10(1):6-20, 2009.

[35] Juan J. Rodriguez, Ludmila I. Kuncheva, and Carlos J. Alonso. Rotation forest: A new classifier ensemble method. IEEE Trans. Pattern Anal. Mach. Intell., 28(10):1619-1630, October 2006.

[36] James A. Russell. Is there universal recognition of emotion from facial expression? a review of the cross-cultural studies. Psychological Bulletin, 115:102-141, 1994.

[37] Lucas Stephane. Cognitive and emotional human models within a multiagent framework. In Proceedings of the International Conference of Human - Computer Interface, Beijing, China, 2007.

[38] Bas R. Steunebrink, Mehdi Dastani, and John-Jules Ch. Meyer. Towards a quantitative model of emotions for intelligent agents. In Dirk Reichardt and Paul Levi, editors, Proceedings of the 2nd Workshop on Emotion and Computing - Current Research and Future Impact, Osnabrck, Germany, 2007.

[39] Ian H. Witten and Eibe Frank. Data Mining: Practical Machine Learning Tools and Techniques. Morgan Kaufmann, San Francisco, 2nd edition, 2005. 